\title{
SunnyTALEN: A Second-Generation TALEN System for Human Genome Editing
}

\author{
Ning Sun, ${ }^{1}$ Zehua Bao, ${ }^{1}$ Xiong Xiong, ${ }^{2}$ Huimin Zhao ${ }^{1,2,3}$ \\ ${ }^{1}$ Department of Biochemistry, University of Illinois at Urbana-Champaign, \\ 600 South Mathews Avenue, Urbana, Illinois 61801; telephone: +217-3332631; \\ fax: +217-3335052; e-mail: zhao5@illinois.edu \\ ${ }^{2}$ Department of Chemical and Biomolecular Engineering, University of Illinois at \\ Urbana-Champaign, 600 South Mathews Avenue, Urbana, Illinois 61801 \\ ${ }^{3}$ Department of Bioengineering, Department of Chemistry, Center for Biophysics and \\ Computational Biology and Institute for Genomic Biology, University of Illinois at \\ Urbana-Champaign, 600 South Mathews Avenue, Urbana, Illinois 61801
}

\begin{abstract}
Transcription activator-like effector nucleases (TALENs) have rapidly emerged as a powerful genome editing tool. The site-specific DNA double-strand breaks generated by TALENs in the human chromosome can induce homologous recombination or non-homologous end joining, resulting in desired genetic modifications. In this study, we report the development of a TALEN variant, SunnyTALEN, with $>2.5$-fold improved genome editing efficacy in human cells. The corresponding scaffold increases the rate of genetic modification at all the 13 tested loci of human genome and is compatible with heterodimer TALEN architectures. This enhanced and high-efficiency TALEN variant represents a novel second-generation TALEN system and has great potential for biological and therapeutic applications.

Biotechnol. Bioeng. 2014;111: 683-691.

(C) 2013 Wiley Periodicals, Inc.
\end{abstract}

KEYWORDS: directed evolution; double-strand break; genome editing; gene therapy; synthetic biology

\section{Introduction}

Genetic modifications of human genomes have a wide range of applications such as investigating human biology, studying disease mechanisms and treating diseases via gene therapies (Cherry and Daley, 2013; Dow and Lowe, 2012). Although

The present address of Ning Sun is Department of Chemistry and Chemical Biology, Harvard University, 12 Oxford Street, Cambridge, MA 02138

Correspondence to: H. Zhao

Contract grant sponsor: Department of Chemical and Biomolecular Engineering at the University of Illinois at Urbana-Champaign (Centennial Chair Professorship)

Received 6 July 2013; Revision received 17 October 2013; Accepted 14 November 2013

Accepted manuscript online 19 November 2013;

Article first published online 11 December 2013 in Wiley Online Library

(http://onlinelibrary.wiley.com/doi/10.1002/bit.25154/abstract).

DOI 10.1002/bit.25154 virus-based transgenesis can modify human genomes efficiently by introduction of exogenous genes, random integration of viral transgenes poses the risk of insertional mutagenesis and carcinogenesis (Check, 2002; Marshall, 1999). In contrast, targeted genome editing or engineering enables researchers to tailor the human genome in a precise manner. To achieve efficient genome editing in human cells, DNA double-strand breaks (DSBs) have to be introduced at the specific sites of the chromosome by custom-designed DNA endonucleases. Subsequent repair of the DSBs by nonhomologous end joining (NHEJ) or homologous recombination can generate desired genomic modifications such as gene disruptions, gene deletions, gene insertions, gene replacements, and chromosome rearrangements (Perez-Pinera et al., 2012; Sun et al., 2012a).

Recently, transcription activator-like effector (TALE) nucleases (TALENs) have emerged as an efficient and versatile tool for genome editing by introduction of chromosomal DNA DSBs (Christian et al., 2010; Joung and Sander, 2013; Miller et al., 2011; Mussolino and Cathomen, 2012; Sun and Zhao, 2013b). TALENs are artificial DNA endonucleases composed of a TALE central repeat domain as the DNA recognition module and a nonspecific FokI nuclease domain as the DNA cleavage module. The TALE central repeat domain comprises a series of tandem repeat units, each of which typically contains 34 residues. Each repeat is used to recognize a single nucleotide and the DNA recognition specificity is determined by the highly variable residues at positions 12 and 13 (e.g., NI recognizes adenine, $\mathrm{HD}$ recognizes cytosine, $\mathrm{NG}$ recognizes thymine, $\mathrm{NH}$ recognizes guanine, and $\mathrm{NN}$ recognizes guanine and adenine) (Boch et al., 2009; Cong et al., 2012; Moscou and Bogdanove, 2009; Streubel et al., 2012). The simple DNA recognition code and the modular nature of the TALE central repeat units enable researchers to tailor DNA recognition specificity with ease and target essentially any 
desired DNA sequence. Therefore, TALENs have been widely applied for genome editing in various species (Sun and Zhao, 2013b). Due to their long recognition sequences (30-50 bp) and high DNA recognition specificity, TALENs exhibit minimal off-target effects in the context of complex human genomes (Ding et al., 2013; Mussolino et al., 2011; Sun et al., 2012b), making them an ideal platform for human genome editing.

First-generation TALEN scaffolds have been described with different N-terminal segments (NTSs) and C-terminal segments (CTSs) flanking the TALE central repeat domains (Kim et al., 2013a; Miller et al., 2011; Mussolino et al., 2011; Sun et al., 2012b). Some of them have been applied for generating human stem cell-based disease models (Ding et al., 2013) and treating human diseases (Choi et al., 2013; Osborn et al., 2013; Sun and Zhao, 2013a), but their efficacy of modifying human genomes is limited in targeting certain loci (Kim et al., 2013a; Reyon et al., 2012). Although a second-generation TALEN platform called GoldyTALEN has been reported with improved genome editing efficacy in zebrafish (Bedell et al., 2012) and livestock (Carlson et al., 2012), its efficacy in modifying human genomes has not been demonstrated. Here, we report the development and application of a high-throughput screening system to improve TALEN activity through a directed evolution strategy. We successfully isolated a TALEN variant, SunnyTALEN, with significantly improved efficacy in modifying human genomes. We demonstrate that the corresponding TALEN scaffold is more active than the GoldyTALEN system in human cells and compatible with previously published obligate heterodimeric FokI nuclease domains. This novel second-generation TALEN system provides a general solution for efficient modifications of human genomes with low toxicities and has great potential in both basic and applied biological sciences.

\section{Materials and Methods}

\section{Materials}

Q5 DNA polymerase, T4 DNA ligase, Antarctic phosphatase, and restriction endonucleases were purchased from New England Biolabs (Beverly, MA). QIAprep Spin Plasmid Miniprep Kit, QIAquick Gel Extraction Kit, and QIAquick PCR Purification Kit were obtained from Qiagen (Valencia, CA). Oligonucleotide primers were obtained from Integrated DNA Technologies (Coralville, IA). All the other reagents unless specified were obtained from Sigma-Aldrich (St. Louis, MO).

\section{Yeast Reporter Strain}

To construct a single-strand annealing enhanced green fluorescent protein (eGFP) reporter, two separated eGFP fragments sharing a 100-bp homologous region were PCR amplified separately, digested with AvrII and $\mathrm{X} b a \mathrm{I}$, respectively, and ligated overnight at $16^{\circ} \mathrm{C}$. The ligation product was subsequently digested with a combination of AvrII and $X b a \mathrm{I}$ and the 870-bp non-cut fragment was gel-purified. The eGFP reporter gene was then cloned into the pRS414 plasmid (New England Biolabs) between the $A D H 1$ promoter and the ADH1 terminator. The centromere sequence of the plasmid was then removed by the PmlI digestion and blunt ligation. This plasmid was then digested with HindIII at the TRP1 gene. The linearized plasmid was transformed into

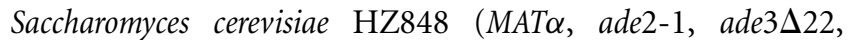
$\Delta$ ura3, his3-11,15, $\operatorname{trp1} 1$ 1, leu2-3,112, and can1-100) using the LiAc/SS carrier DNA/PEG method (Gietz and Schiestl, 2007) and stably integrated into the chromosome at the TRP1 site. The yeast reporter strain was obtained by selection of the transformed yeast cells on plates containing synthetic complete medium lacking tryptophan with $2 \%$ glucose at $30^{\circ} \mathrm{C}$. The integration was confirmed by PCR amplification and DNA sequencing of the targeted genomic region.

\section{Library Creation}

The pRS415 yeast expression vector encoding the AvrXa10 TALEN that has been described previously (Sun et al., 2012b) was used as the template for mutagenic PCR. The sequence encoding the CTS and FokI nuclease domain was mutagenized using the GeneMorph II Random Mutagenesis Kit (Stratagene, La Jolla, CA) according to the manufacturer's instruction. The following primers were used for the amplification: EP-FokI-Far-for $5^{\prime}$-ttg ttg ccc agt tat ctc gc- $3^{\prime}$ and EP-FokI-Far-rev $5^{\prime}$-cgt gaa act tcg aac act gtc-3'. Two libraries with a low mutation rate $(0-4.5$ mutations $/ \mathrm{kbp})$ and a medium mutation rate (4.5-9 mutations/kbp) were amplified and gel-purified, respectively. The TALEN expression plasmid was digested with AatII and SalI and gel purified to remove the sequences encoding the wild-type CTS and FokI domain. The two gene libraries were mixed and cotransformed with the linearized TALEN expression plasmids at an approximately 13:1 insert:vector molar ratio into the yeast reporter strain using the LiAc/SS carrier DNA/PEG method (Gietz and Schiestl, 2007). Through yeast homologous recombination, the pRS415 plasmid library was created containing $\sim 2 \times 10^{5}$ transformants.

\section{High-Throughput Screening}

After transformation, the yeast reporter strain carrying the library of TALEN variants was recovered at $30^{\circ} \mathrm{C}$ with shaking for $1 \mathrm{~h}$ in YPA medium (1\% yeast extract, $2 \%$ peptone, and $0.01 \%$ adenine hemisulfate) with $2 \%$ glucose. The cells were then centrifuged and resuspended in synthetic complete medium lacking leucine and tryptophan (SC-Leu-Trp) with $2 \%$ raffinose for growing at $30^{\circ} \mathrm{C}$ with shaking overnight. TALEN expression was induced by culturing cells in YPA medium with $0.002-2 \%$ galactose at $30^{\circ} \mathrm{C}$ for $1-6 \mathrm{~h}$ (the induction conditions for each cycle of screening are listed in Supplementary Fig. 2). The cells were then centrifuged and resuspended in SC-Leu-Trp liquid medium with $2 \%$ glucose 
at $30^{\circ} \mathrm{C}$ with shaking overnight. On the next day, cells were analyzed on a BD FACS Aria III cell sorting system (BD Biosciences, San Jose, CA) and 20,000-100,000 eGFPpositive cells were collected. After 2 days' growth at $30^{\circ} \mathrm{C}$ in SC-Leu-Trp liquid medium with $2 \%$ glucose, plasmids from the sorted cells were extracted using Zymoprep Yeast Plasmid Miniprep II Kit (Zymo Research, Orange, CA). Sequences encoding the CTS and FokI domain were PCR amplified from the plasmids by Q5 DNA polymerase and gel purified for the next cycle of screening.

\section{A Modified Surrogate Reporter System}

A TALEN target site within the sickle human beta-globin $\left(H B B^{S}\right)$ gene was inserted between the monomeric red fluorescent protein $(m R F P)$ gene and the eGFP gene by overlap extension PCR. The fusion gene was cloned into the pLNCX2 retroviral vector (BD Clontech, Palo Alto, $\mathrm{CA}$ ) in the presence of the cytomegalovirus $(C M V)$ promoter and stably integrated into the genome of Hela cells through retroviral transduction according to the manufacturer's protocol. Transfected cells were selected in $500 \mu \mathrm{g} / \mathrm{mL}$ G418 for 2 weeks. The TALEN genes along with a FLAG tag sequence and a SV40 nucleus localization signal added to the $\mathrm{N}$-terminus were cloned into the pCMV5 mammalian expression vector (Andersson et al., 1989) through the KpnI and SalI sites.

Reporter Hela cells were routinely maintained in the modified Eagle's medium (MEM) supplemented with 10\% fetal bovine serum (FBS; Hyclone, Logan, UT). Cells were seeded in 24-well plates at a density of $5 \times 10^{4}$ per well. After $24 \mathrm{~h}$, reporter cells were transfected with certain amounts of TALEN expression plasmids using FuGene HD transfection reagent (Promega, Madison, WI) under conditions specified by the manufacturer. For each transfection, the overall plasmid amount was made to be $500 \mathrm{ng}$ by adding the empty pCMV5 plasmid. Cells were trypsinized from their culturing plates $48 \mathrm{~h}$ after transfection and resuspended in $300 \mu \mathrm{L}$ phosphate-buffered saline (PBS) for flow cytometry analysis. Twenty thousand cells were analyzed by a BD LSRII flow cytometer (BD Biosciences) to quantify the eGFP-positive cells.

\section{Western Blot Analysis}

Hela cells in 12-well plates were harvested $24 \mathrm{~h}$ after transfection with pCMV5-TALEN-HBB-R plasmid (Sun et al., 2012b). Cells were collected by centrifugation, washed with PBS, and resuspended in $40 \mu \mathrm{L}$ whole cell lysis buffer (1 M Tris-HCl, pH 6.8, 20\% sodium dodecyl sulfate, and $0.1 \mathrm{M}$ dithiothreitol). Proteins were resolved by 4-20\% MiniProtean TGX Precast Gel (Bio-Rad Laboratories, Hercules, CA), transferred onto a nitrocellulose membrane, blocked for $1 \mathrm{~h}$ with Tris-buffered saline/0.05\% Tween 20 containing 5\% nonfat milk, followed by incubation with anti-FLAG tag (1:500) and anti- $\alpha$-tubulin $(1: 10,000)$ antibodies at $4^{\circ} \mathrm{C}$ overnight. After incubation with anti-mouse horseradish peroxidase-conjugated secondary antibody (1:25,000; Gene-
Script, Piscataway, NJ) for $1 \mathrm{~h}$, bands were visualized using SuperSignal West Pico Chemiluminescent Substrate (Pierce, Rockford, IL).

\section{Results}

\section{Development of a High-Throughput Screening System}

To improve the performance of the existing TALEN technology using directed evolution, we developed a high throughput screening system in $S$. cerevisiae. This system employs an eGFP-based single-strand annealing reporter construct that is integrated into the yeast genome. The reporter construct contains a divided $e G F P$ gene harboring two AvrXa10 TALE binding sites in a tail-to-tail orientation (Supplementary Table I). The DSB generated by the AvrXa10 TALEN homodimer can induce single-strand annealing of the two truncated $e G F P$ DNA fragments and reconstitute a complete and functional eGFP gene (Fig. 1A). Therefore, this system couples the enzymatic DNA cleavage activity with the fluorescent signal of host cells, and enables high-throughput screening of TALEN mutants with improved genome editing
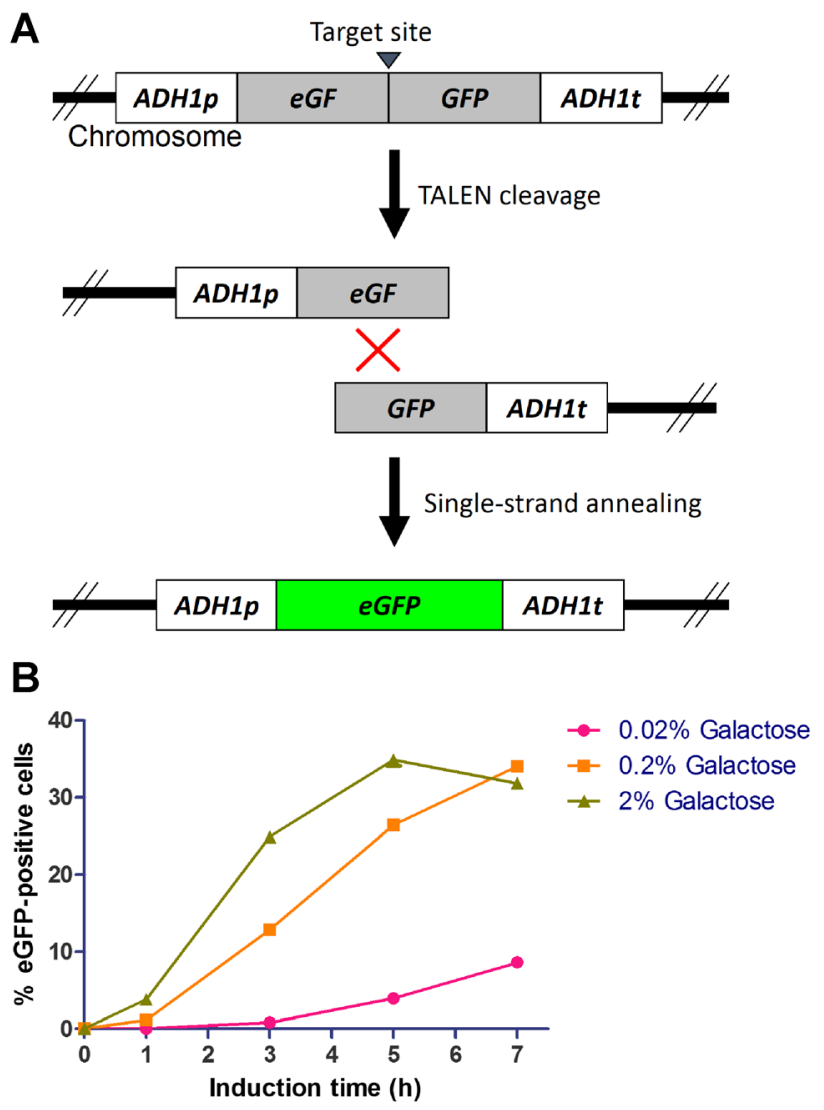

Figure 1. An eGFP-based single-strand annealing reporter system in yeast. $\mathbf{A}$ : Schematic of the yeast reporter system. $A D H 1 p$, promoter of the $A D H 1$ gene; $A D H 1 t$ transcription terminator of the $A D H 1$ gene; $e G F, 5^{\prime}$-fragment of the eGFP gene; and GFP, $3^{\prime}$-fragment of the eGFP gene. B: Activities of the WT TALEN in the yeast reporter system under different induction conditions. 
efficacy. The TALEN gene expression is under the control of a galactose-inducible promoter, thus the selection pressure of directed evolution can be adjusted by galactose concentration in the growth medium and incubation time during induction (Fig. 1B).

\section{Directed Evolution of TALENs With Improved Activity}

Using a TALEN scaffold bearing a 207 aa NTS and a 63 aa CTS (hereinafter referred to as WT) (Sun et al., 2012b) as a template, we randomly mutagenized the sequences encoding the CTS and the FokI nuclease domain using error-prone PCR and constructed a library of mutants in vivo by gap repair homologous recombination in yeast (Supplementary Fig. 1). TALEN expression was induced by galactose and the eGFP-positive cells were collected by fluorescenceactivated cell sorting (FACS). The sorted cells were pooled together and plasmids from the pool were isolated. The regions encoding the CTS and FokI nuclease domain of TALEN genes were PCR-amplified from the plasmids and subjected to another round of screening. This process was continued in an iterative fashion until the TALEN mutants with improved genome editing activity were enriched and identified (Fig. 2). We gradually increased the selection stringency by decreasing the galactose concentration and induction time during the enrichment (Supplementary Fig. 2). After two rounds of mutagenesis and evolution, 14 TALEN mutants with improved activity were generated (Table I, Fig. 3 and Supplementary Fig. 3). The

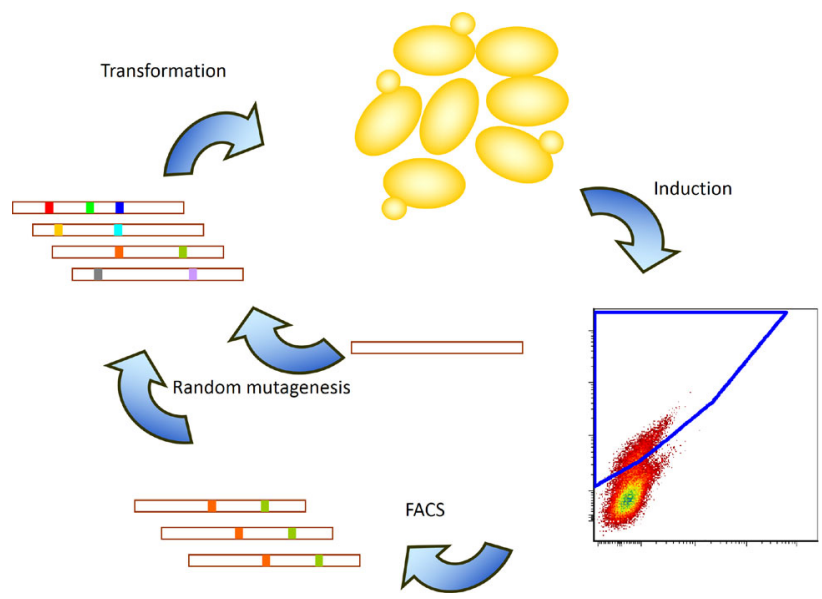

Figure 2. Schematic representation of the directed evolution approach in yeast for isolating novel TALEN variants.

most active mutant (2S3C3) has greater than sevenfold improvement in yeast genome modification compared to WT, whereas GoldyTALEN and the TALEN bearing a Sharkey domain (a FokI domain variant with S35P and K58E mutations) (Guo et al., 2010) only exhibit $<3.5$-fold increase compared to WT.

Table I. Information and in vivo activities of TALEN variants.

\begin{tabular}{|c|c|c|c|}
\hline \multirow[b]{2}{*}{ Label } & \multirow[b]{2}{*}{ Mutation $^{\mathrm{a}}$} & \multicolumn{2}{|c|}{ Relative activity (fold of WT) ${ }^{\mathrm{b}}$} \\
\hline & & Yeast $^{\mathrm{c}}$ & Human cells ${ }^{\mathrm{d}}$ \\
\hline WT & - & $1.00 \pm 0.11$ & $1.00 \pm 0.05$ \\
\hline Sharkey & S35P, K58E & $2.38 \pm 0.23$ & $1.01 \pm 0.02$ \\
\hline Goldy $^{\mathrm{e}}$ & $\mathrm{L}(-33) \mathrm{M}, \mathrm{I}(-16) \mathrm{V}, \mathrm{P}(-11) \mathrm{G}$ & $3.24 \pm 0.15$ & $0.80 \pm 0.06$ \\
\hline S4C5 & $\mathrm{P}(-11) \mathrm{T}, \mathrm{H}(-6) \mathrm{Q}, \mathrm{V} 52 \mathrm{I}$ & $3.49 \pm 0.14$ & $2.08 \pm 0.10$ \\
\hline S4C9 & $\mathrm{P}(-11) \mathrm{H}, \mathrm{L} 182 \mathrm{~L}, \mathrm{R} 186 \mathrm{R}$ & $2.90 \pm 0.32$ & $2.26 \pm 0.11$ \\
\hline S4C10 & K58R, N144K & $1.30 \pm 0.10$ & $0.40 \pm 0.03$ \\
\hline $\mathrm{S} 7 \mathrm{C} 1$ & $\mathrm{P}(-11) \mathrm{R}, \mathrm{I} 82 \mathrm{I}, \mathrm{H} 115 \mathrm{H}, \mathrm{N} 153 \mathrm{~K}, \mathrm{~L} 168 \mathrm{~L}, \mathrm{~L} 182 \mathrm{~L}$ & $4.40 \pm 0.16$ & $1.11 \pm 0.03$ \\
\hline S7C6 & $\mathrm{P}(-11) \mathrm{H}$ & $4.11 \pm 0.23$ & $2.26 \pm 0.03$ \\
\hline S7C7 & S5S, I175I, K176Q, L182L & $2.83 \pm 0.17$ & $1.62 \pm 0.08$ \\
\hline S7C10 & K58E, I70I, L182L, E193V & $1.76 \pm 0.22$ & $0.10 \pm 0.01$ \\
\hline Sunny & P(-11)H, S35P, K58E & $4.13 \pm 0.27$ & $2.69 \pm 0.43$ \\
\hline 2S3C3 & $\mathrm{P}(-11) \mathrm{H}, \mathrm{S} 35 \mathrm{P}, \mathrm{K} 51 \mathrm{E}, \mathrm{K} 58 \mathrm{E}$ & $7.26 \pm 0.38$ & $2.21 \pm 0.14$ \\
\hline $2 \mathrm{~S} 3 \mathrm{C} 11$ & $\mathrm{P}(-11) \mathrm{H}, \mathrm{S} 35 \mathrm{P}, \mathrm{K} 58 \mathrm{E}, \mathrm{A} 87 \mathrm{~A}, \mathrm{~F} 134 \mathrm{Y}$ & $6.85 \pm 0.42$ & $0.91 \pm 0.02$ \\
\hline $2 \mathrm{~S} 3 \mathrm{C} 12$ & L(-33)L, P(-11)H, L18L, S35P, K58E, K123N & $4.91 \pm 0.40$ & $1.96 \pm 0.09$ \\
\hline $2 \mathrm{~S} 3 \mathrm{C} 18$ & $\mathrm{P}(-11) \mathrm{H}, \mathrm{E}(-10) \mathrm{K}, \mathrm{L} 7 \mathrm{~L}, \mathrm{~S} 35 \mathrm{P}, \mathrm{K} 58 \mathrm{E}, \mathrm{I} 70 \mathrm{M}, \mathrm{P} 76 \mathrm{~L}, \mathrm{~V} 81 \mathrm{~V}$ & $6.20 \pm 0.57$ & $1.69 \pm 0.14$ \\
\hline $2 \mathrm{~S} 6 \mathrm{C} 1$ & $\mathrm{P}(-11) \mathrm{H}, \mathrm{N} 34 \mathrm{D}, \mathrm{K} 58 \mathrm{E}$ & $5.88 \pm 0.56$ & $1.10 \pm 0.05$ \\
\hline $2 \mathrm{~S} 8 \mathrm{C} 6$ & $\mathrm{P}(-11) \mathrm{H}, \mathrm{E}(-10) \mathrm{K}, \mathrm{S} 35 \mathrm{P}, \mathrm{K} 58 \mathrm{E}$ & $5.67 \pm 0.47$ & $2.03 \pm 0.19$ \\
\hline
\end{tabular}

\footnotetext{
${ }^{\mathrm{a}}$ The residue numbering is indicated in Supplementary Figure 1B. Silent mutations are shown in gray.

${ }^{\mathrm{b}}$ The activity of WT was normalized to 1 . Values represent the average ( \pm standard deviation) of at least three independent experiments.

${ }^{c}$ Activities in yeast were measured when the TALEN expression was induced in the culture medium containing $0.02 \%$ galactose for $4 \mathrm{~h}$.

${ }^{\mathrm{d} A c t i v i t i e s ~ i n ~ h u m a n ~ c e l l s ~ w e r e ~ m e a s u r e d ~ i n ~ t h e ~ m o d i f i e d ~ s u r r o g a t e ~ r e p o r t e r ~ s y s t e m ~(F i g . ~ 4 A) ~ i n ~ a ~ d o s e-l i m i t i n g ~ c o n d i t i o n ~(~} 32 \mathrm{ng}$ transfected TALEN expression plasmids per $10^{5}$ cells).

${ }^{\mathrm{e}}$ GoldyTALEN has a shorter and mutated NTS compared with all the other TALENs. Complete sequence is shown in Supplementary Figure 11.
} 


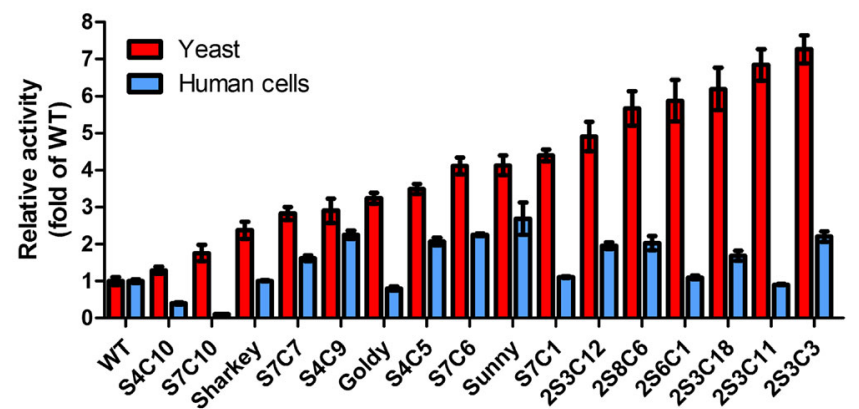

Figure 3. Relative activities of TALEN variants in yeast and human cells. Activities in yeast were measured when the TALEN expression was induced in the culture medium containing $0.02 \%$ galactose for $4 \mathrm{~h}$. Activities in human cells were measured in the modified surrogate reporter system (Fig. $4 \mathrm{~A}$ ) in a dose-limiting condition $(32 \mathrm{ng}$ transfected TALEN expression plasmids per 105 cells). The activity of WT was normalized to 1 . Error bars indicate standard deviation of at least three independent experiments. The mutations and numeric values are shown in Table I.

A
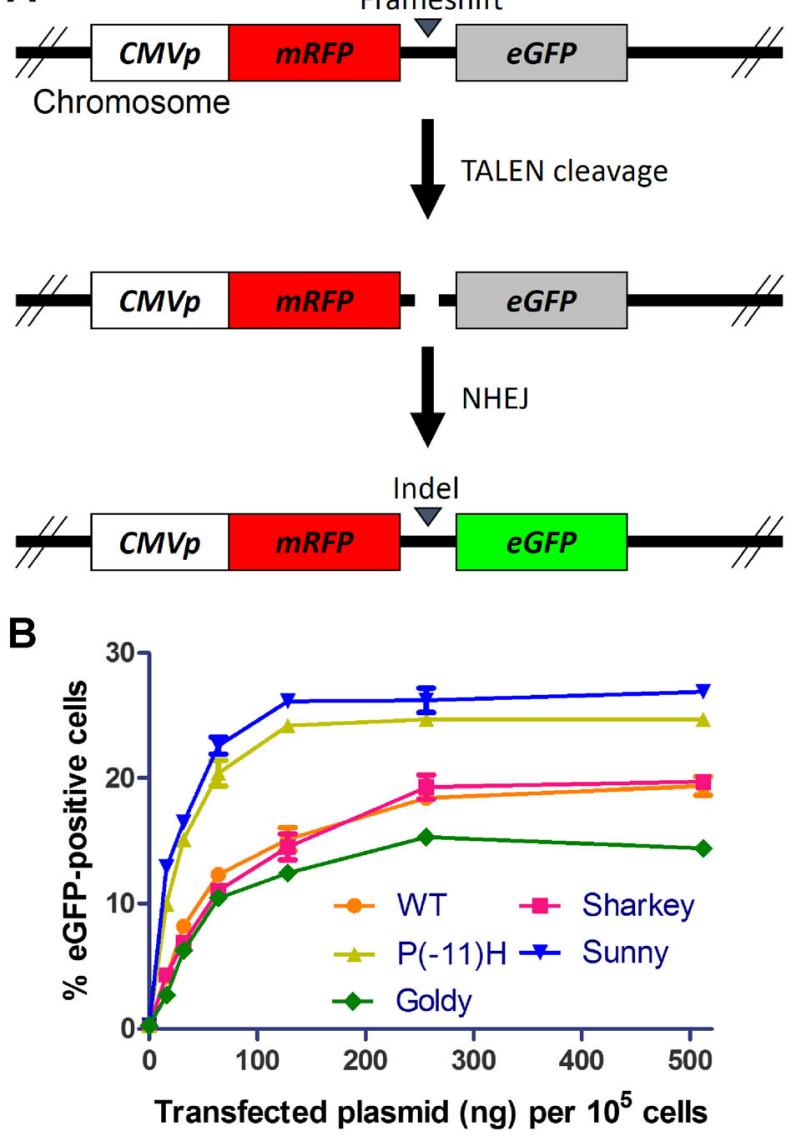

Figure 4. Performance of TALEN variants in a modified surrogate reporter system. A: Schematic of the modified surrogate reporter system in Hela cells. CMVp, CMV promoter. B: Dose-response curve using titrations of different TALEN expression plasmids as tested in the modified surrogate reporter system in Hela cells. Error bars indicate standard deviation of three independent experiments.

\section{Isolation and Characterization of the SunnyTALEN Scaffold}

To test the efficacy of the isolated TALEN mutants in human cells, we modified a surrogate reporter system (Kim et al., 2011) to rapidly and quantitatively gauge the potential of TALEN-driven genome editing (Fig. 4A). The reporter construct encoding a mRFP-eGFP fusion protein was stably integrated into the genome of Hela cells. A TALEN target site within the $H B B^{S}$ gene locus (Supplementary Table I) (Sun et al., 2012b) was inserted between the mRFP and eGFP genes, making the eGFP gene out of frame. In response to the TALEN-induced DSBs, certain insertions and deletions (indels) caused by NHEJ-mediated mutagenesis will make the eGFP gene in frame and restore eGFP function. Each TALEN mutant was under the control of a $C M V$ promoter, and genome editing activity was measured by flow cytometry following transient expression. Although the TALEN bearing a Sharkey domain and GoldyTALEN showed similar or lower genome editing efficacy compared to WT, one TALEN mutant (referred to as SunnyTALEN) has $>2.5$-fold improvement compared to WT (Table I, Fig. 3 and Supplementary Fig. 4). SunnyTALEN contains a single P(-11) $\mathrm{H}$ mutation on the CTS and a Sharkey domain. We performed titrations of the expression plasmids of different TALEN architectures at various amounts and obtained a dose-response curve (Fig. 4B). SunnyTALEN consistently yielded more eGFP-positive cells compared to the other TALEN variants. The $\mathrm{P}(-11) \mathrm{H}$ mutation of SunnyTALEN serves as a major contributor to its improved efficacy without changing steady state level of protein. The S35P and K58E mutations on the Sharkey domain increase activity marginally, which can be explained by the increased steady state level of protein (Figs. 4B and 5). The lower protein level of GoldyTALEN is consistent with its lower efficacy in human cells than WT. In addition, cells transfected with TALEN variants were subjected to the immunofluorescent analysis for cellular localization. The results showed that all TALEN variants, which contain an $\mathrm{N}$-terminal nuclear localization signal, were predominantly localized in the nuclei (Supplementary Fig. 5).

\section{Application of the SunnyTALEN Scaffold for Human Genome Editing}

To further explore the general applicability of the SunnyTALEN scaffold in modifying human genomes, we generated TALENs against 12 additional genomic loci of human embryonic kidney (HEK)293 cells. Using the same TALE DNA binding domains in the SunnyTALEN scaffold, we observed increased rate of gene modification at all the targeted loci compared to WT, with the increased indels ranging from $4 \%$ to $33 \%$ in response to NHEJ-mediated mutagenesis (Fig. 6A and Supplementary Fig. 6). The SunnyTALEN scaffold also showed higher rate of gene modification compared to the WT TALEN with the Sharkey mutations or with the $\mathrm{P}(-11) \mathrm{H}$ substitution (Supplementary Fig. 7). Sequencing analysis of the two targeted loci indicated 


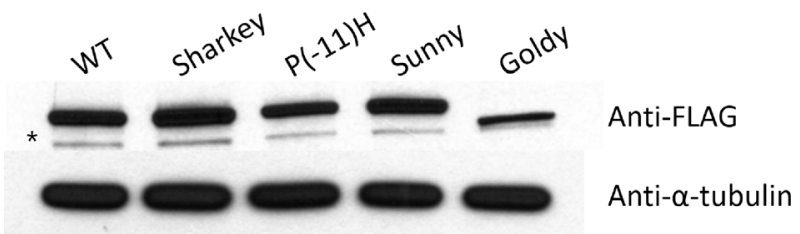

Figure 5. Western blot analysis of TALEN variants in human cells. All the TALEN variants contained a FLAG tag at the N-terminus. $\alpha$-Tubulin was used as a loading control. An asterisk indicates non-specific bands.

that SunnyTALENs induced more small deletions than insertions (Supplementary Fig. 8), with the mutation signatures similar to the first-generation TALENs (Kim et al., 2013b) and GoldyTALENs (Bedell et al., 2012). We also explored whether the rates of TALEN-induced homologous recombination could be enhanced using an eGFP gene conversion assay (Supplementary Fig. 9A) (Sun et al., 2012b). In the linear range of the dose-response curve, we observed a $>2$-fold increase in homology-directed gene repair using the SunnyTALEN scaffold over WT (Supplementary Fig. 9B), which paralleled the increase observed in NHEJ-mediated gene disruption levels (Fig. 4B).

To prevent the formation of unexpected cleavage-competent homodimers, obligate heterodimeric FokI domains have been used in TALENs to reduce off-target effects and cytotoxicities (Cade et al., 2012; Huang et al., 2011). To investigate the compatibility of the SunnyTALEN scaffold with the obligate heterodimeric FokI domains, we introduced the ELD:KKR FokI pair (ELD denotes Q103E, I116L, and N113D mutations; KKR denotes E107K, I155K, and H154R mutations) (Doyon et al., 2011) that preferentially heterodimerize into the SunnyTALEN scaffold and compared the genome editing efficacy with the WT counterpart in the modified surrogate reporter system (Fig. 4A). The pairwise analysis of these architectures showed that the TALEN
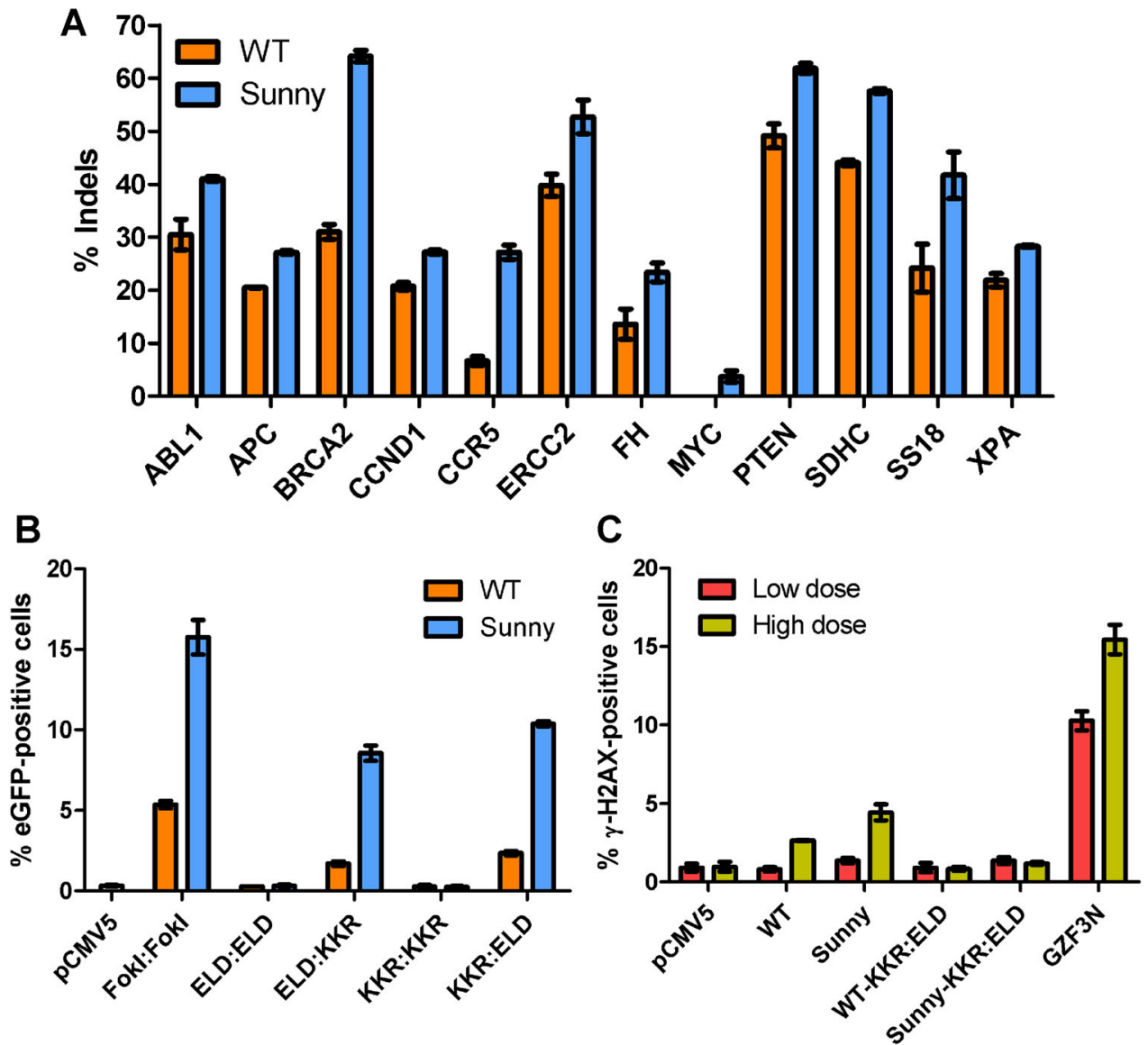

Figure 6. Characteristics of the SunnyTALEN platform in human genome editing. A: The SunnyTALEN scaffold improved NHEJ-mediated gene disruptions at 12 genomic loci in HEK293 cells. Representative gel images of the SURVEYOR nuclease assays are shown in Supplementary Figure 6. B: The SunnyTALEN scaffold is compatible with the obligate heterodimeric Fokl nuclease domains. Activities were measured in the modified surrogate reporter system (Fig. 4A) in a dose-limiting condition (32 ng transfected TALEN expression plasmids per $10^{5}$ cells). Error bars indicate standard deviation of three independent experiments. Empty vector pCMV5 served as a negative control. C: Nuclease-associated cytotoxicities of TALENs. Low dose indicates 32-ng TALEN expression plasmids were transfected per $10^{5}$ cells. High dose indicates $256 \mathrm{ng}$ TALEN expression plasmids were transfected per $10^{5}$ cells. A reported toxic zinc finger nuclease (GZF3N) served as a positive control (Szczepek et al., 2007). Error bars indicate standard deviation of three independent experiments. 
heterodimers with SunnyTALEN scaffold had $>4.4$-fold improvement at stimulating mutagenesis compared to the WT TALEN heterodimers (Fig. 6B). None of the ELD:ELD or KKR:KKR TALEN dimers were observed to be cleavage competent. To assess the nuclease-associated toxicity, we monitored the phosphorylation of histone $\mathrm{H} 2 \mathrm{AX}(\boldsymbol{\gamma}-\mathrm{H} 2 \mathrm{AX})$, which rapidly responds to chromosomal DSBs and can be used to measure genome-wide DNA damage levels. Although the SunnyTALEN scaffold slightly increased $\gamma$-H2AX level over WT, the introduction of the obligate heterodimeric FokI domains reduced $\gamma-\mathrm{H} 2 \mathrm{AX}$ staining to the background level (Fig. 6C). Based on the prediction by TAL Effector Nucleotide Targeter 2.0 (Doyle et al., 2012), a highly similar sequence within the $H B D$ gene that shares only four mismatches compared with the designed TALEN binding site was identified (Supplementary Fig. 10A). Modest off-target cleavage at this site was observed using the SunnyTALEN scaffold at high dose (Supplementary Fig. 10B).

\section{Discussion}

In this study, we developed a powerful yeast-based screening system to improve TALEN activity. This system couples enzymatic DNA cleavage with GFP signal of yeast cells and enables directed evolution of TALENs with improved genome editing efficacy. The GFP-positive cells can be isolated by FACS, which enables high-throughput screening of large libraries of mutants with single cell resolution and low false-positive rate. Although applied here in enhancing TALEN activity, this system can be easily adapted to engineer other rare-cutting DNA endonucleases such as homing endonucleases (or meganucleases) (Silva et al., 2011), zinc finger nucleases (ZFNs) (Carroll, 2011), and newly developed clustered regularly interspaced short palindromic repeats (CRISPR)-Cas system (Cong et al., 2013; Hwang et al., 2013; Jiang et al., 2013; Jinek et al., 2013; Mali et al., 2013), for the purpose of increasing catalytic activities or altering DNA recognition specificities.

In the SunnyTALEN scaffold, the $\mathrm{P}(-11) \mathrm{H}$ mutation on the CTS plays a major role in activity improvement. Because structural information is not available to visualize that residue, we have built structural models of WT and SunnyTALEN scaffolds with 63 aa CTSs to study the molecular mechanism. Structural models indicate the substitution of a histidine for a proline introduces an additional hydrogen bond between the CTS of the SunnyTALEN scaffold and the DNA backbone, which might increase catalytic activity by positioning the FokI nuclease domain closer to the DNA substrate or to the FokI domain of the other TALEN monomer (Fig. 7). More detailed structural studies will be needed to confirm this hypothesis. In addition to the isolation of a high-efficiency TALEN system, our directed evolution endeavor identified "hot spot" residues (e.g., $\mathrm{P}(-11), \mathrm{E}(-10), \mathrm{N} 34, \mathrm{~S} 35, \mathrm{~K} 51, \mathrm{~K} 58, \mathrm{~F} 134$, and K176) that are critical for the catalytic activity or protein solubility of TALENs (Table I), which provides an important insight for the further improvement of TALEN system by rational protein design or saturation mutagenesis.

Using $\gamma-\mathrm{H} 2 \mathrm{AX}$ as a marker of chromosomal DNA damage, we measured the nuclease-associated genotoxicities in human cells. Even though the increased genome editing efficacy of the SunnyTALEN scaffold is accompanied by increased DNA damage, $\gamma$-H2AX level can be reduced by lowering the TALEN dose or introducing obligate
WT

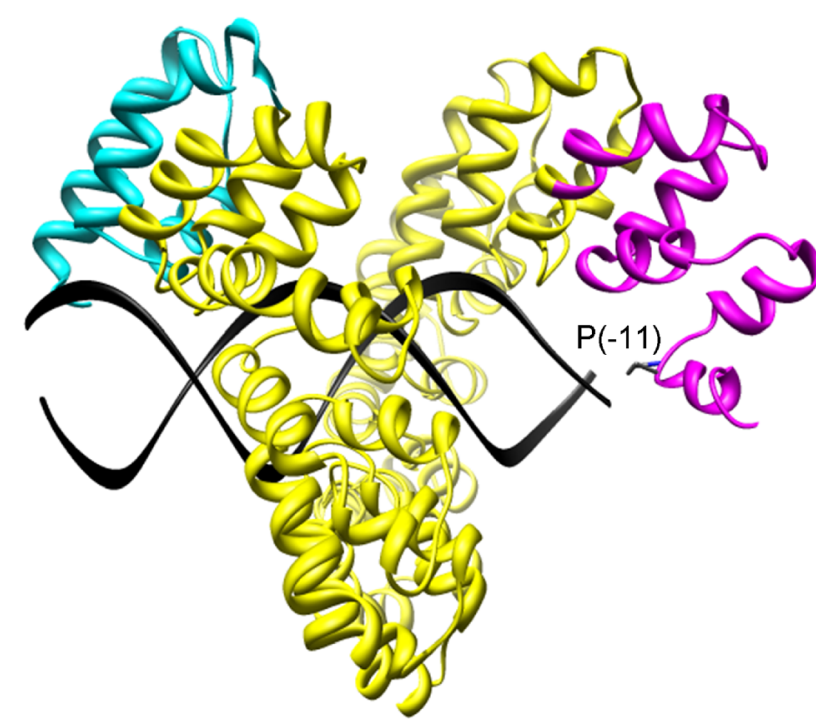

Sunny

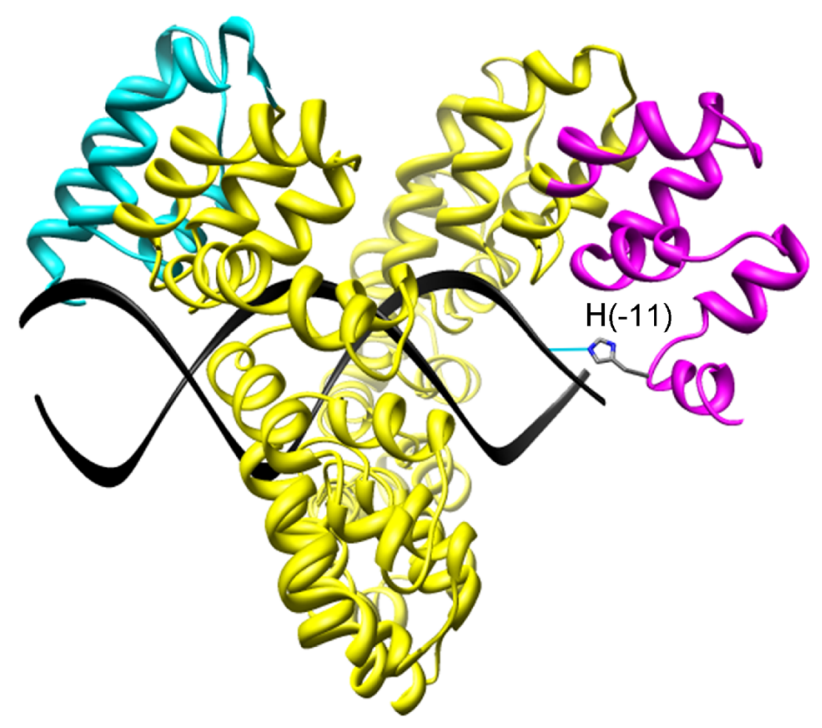

Figure 7. Structural models of the WT and SunnyTALEN scaffolds. DNA substrates are shown in black ribbons. The NTSs are shown in cyan. The central repeat units are shown in yellow. The CTSs are shown in magenta. A hydrogen bond is shown in cyan. 
heterodimeric FokI nuclease domains (Fig. 6C). Because of the high efficiency of the SunnyTALEN scaffold, it can generate sufficient genetic modifications at dose-limiting conditions without causing detectable genotoxicities (Figs. 4B and 6C). Off-target cleavage is dependent on protein concentration as demonstrated for ZFNs (Pattanayak et al., 2011). Here we also showed that lowering the dose of SunnyTALENs decreased the cleavage at an off-target site (Supplementary Fig. 10B). Therefore, it is suggested to optimize the dose of SunnyTALENs for different human cell lines to achieve efficient genome editing with low toxicity. To avoid the off-target events generated by homodimeric ZFNs, obligate heterodimer mutations were introduced at the dimer interface of the FokI cleavage domain to prevent homodimerization based on electrostatic and hydrophobic interactions (Doyon et al., 2011; Miller et al., 2007; Szczepek et al., 2007). Similar principle was applied to TALENs and successfully reduced off-target cleavage and relieved toxicity (Cade et al., 2012). We show here that the incorporation of obligate heterodimeric FokI domains completely abolished the formation of cleavage-competent SunnyTALEN homodimers, resulting in reduced genotoxicity (Fig. 6B and C).

In conclusion, we isolated the high-efficiency SunnyTALEN scaffold using a directed evolution strategy. The SunnyTALEN scaffold is portable to many TALE DNA binding domains and compatible with heterodimer TALEN architectures. Although deployed here in yeast and human cells, the SunnyTALEN scaffold could be effective in various organisms and cell types. In addition to the GoldyTALEN scaffold that enables efficient genome modifications in zebrafish and livestock, the SunnyTALEN scaffold serves as a novel second-generation TALEN system and will aid in a variety of applications in medicine and biology.

This work was supported by the Centennial Chair Professorship (H.Z.) in the Department of Chemical and Biomolecular Engineering at the University of Illinois at Urbana-Champaign. We thank the University of Illinois Flow Cytometry Facility for assistance with flow cytometry and FACS.

\section{References}

Andersson S, Davis DL, Dahlback H, Jornvall H, Russell DW. 1989. Cloning, structure, and expression of the mitochondrial cytochrome P-450 sterol 26-hydroxylase, a bile acid biosynthetic enzyme. J Biol Chem 264(14):8222-8229.

Bedell VM, Wang Y, Campbell JM, Poshusta TL, Starker CG, Krug RG II, Tan W, Penheiter SG, Ma AC, Leung AY, Fahrenkrug SC, Carlson DF, Voytas DF, Clark KJ, Essner JJ, Ekker SC. 2012. In vivo genome editing using a high-efficiency TALEN system. Nature 491(7422):114-118.

Boch J, Scholze H, Schornack S, Landgraf A, Hahn S, Kay S, Lahaye T, Nickstadt A, Bonas U. 2009. Breaking the code of DNA binding specificity of TAL-type III effectors. Science 326(5959):1509-1512.

Cade L, Reyon D, Hwang WY, Tsai SQ, Patel S, Khayter C, Joung JK, Sander JD, Peterson RT, Yeh JR. 2012. Highly efficient generation of heritable zebrafish gene mutations using homo- and heterodimeric TALENs. Nucleic Acids Res 40(16):8001-8010.

Carlson DF, Tan W, Lillico SG, Stverakova D, Proudfoot C, Christian M, Voytas DF, Long CR, Whitelaw CB, Fahrenkrug SC. 2012. Efficient TALEN-mediated gene knockout in livestock. Proc Natl Acad Sci USA 109(43):17382-17387.
Carroll D. 2011. Genome engineering with zinc-finger nucleases. Genetics 188(4):773-782.

Check E. 2002. A tragic setback. Nature 420(6912):116-118.

Cherry AB, Daley GQ. 2013. Reprogrammed cells for disease modeling and regenerative medicine. Annu Rev Med 64:277-290.

Choi SM, Kim Y, Shim JS, Park JT, Wang RH, Leach SD, Liu JO, Deng CX, Ye Z, Jang YY. 2013. Efficient drug screening and gene correction for treating liver disease using patient-specific stem cells. Hepatology 57(6):2458-2468.

Christian M, Cermak T, Doyle EL, Schmidt C, Zhang F, Hummel A, Bogdanove AJ, Voytas DF. 2010. Targeting DNA double-strand breaks with TAL effector nucleases. Genetics 186(2):757-761.

Cong L, Zhou R, Kuo YC, Cunniff M, Zhang F. 2012. Comprehensive interrogation of natural TALE DNA-binding modules and transcriptional repressor domains. Nat Commun 3:968.

Cong L, Ran FA, Cox D, Lin S, Barretto R, Habib N, Hsu PD, Wu X, Jiang W, Marraffini LA, Zhang F. 2013. Multiplex genome engineering using CRISPR/Cas systems. Science 339(6121):819-823.

Ding Q, Lee YK, Schaefer EA, Peters DT, Veres A, Kim K, Kuperwasser N, Motola DL, Meissner TB, Hendriks WT, Trevisan M, Gupta RM, Moisan A, Banks E, Friesen M, Schinzel RT, Xia F, Tang A, Xia Y, Figueroa E, Wann A, Ahfeldt T, Daheron L, Zhang F, Rubin LL, Peng LF, Chung RT, Musunuru K, Cowan CA. 2013. A TALEN genome-editing system for generating human stem cell-based disease models. Cell Stem Cell 12(2):238-251.

Dow LE, Lowe SW. 2012. Life in the fast lane: Mammalian disease models in the genomics era. Cell 148(6):1099-1109.

Doyle EL, Booher NJ, Standage DS, Voytas DF, Brendel VP, Vandyk JK, Bogdanove AJ. 2012. TAL effector-nucleotide targeter (TALE-NT) 2.0: Tools for TAL effector design and target prediction. Nucleic Acids Res 40 (Web Server issue):W117-W122.

Doyon Y, Vo TD, Mendel MC, Greenberg SG, Wang J, Xia DF, Miller JC, Urnov FD, Gregory PD, Holmes MC. 2011. Enhancing zinc-fingernuclease activity with improved obligate heterodimeric architectures. Nat Methods 8(1):74-79.

Gietz RD, Schiestl RH. 2007. High-efficiency yeast transformation using the LiAc/SS carrier DNA/PEG method. Nat Protoc 2(1): 31-34.

Guo J, Gaj T, Barbas CF III. 2010. Directed evolution of an enhanced and highly efficient FokI cleavage domain for zinc finger nucleases. J Mol Biol 400(1):96-107.

Huang P, Xiao A, Zhou M, Zhu Z, Lin S, Zhang B. 2011. Heritable gene targeting in zebrafish using customized TALENs. Nat Biotechnol 29(8):699-700.

Hwang WY, Fu Y, Reyon D, Maeder ML, Tsai SQ, Sander JD, Peterson RT, Yeh JR, Joung JK. 2013. Efficient genome editing in zebrafish using a CRISPR-Cas system. Nat Biotechnol 31(3):227-229.

Jiang W, Bikard D, Cox D, Zhang F, Marraffini LA. 2013. RNA-guided editing of bacterial genomes using CRISPR-Cas systems. Nat Biotechnol 31(3):233-239.

Jinek M, East A, Cheng A, Lin S, Ma E, Doudna J. 2013. RNA-programmed genome editing in human cells. Elife 2:e00471.

Joung JK, Sander JD. 2013. TALENs: A widely applicable technology for targeted genome editing. Nat Rev Mol Cell Biol 14(1):49-55.

Kim H, Um E, Cho SR, Jung C, Kim H, Kim JS. 2011. Surrogate reporters for enrichment of cells with nuclease-induced mutations. Nat Methods 8(11):941-943.

Kim Y, Kweon J, Kim A, Chon JK, Yoo JY, Kim HJ, Kim S, Lee C, Jeong E, Chung E, Kim D, Lee MS, Go EM, Song HJ, Kim H, Cho N, Bang D, Kim S, Kim JS. 2013a. A library of TAL effector nucleases spanning the human genome. Nat Biotechnol 31(3):251-258.

Kim Y, Kweon J, Kim JS. 2013b. TALENs and ZFNs are associated with different mutation signatures. Nat Methods 10(3):185.

Mali P, Yang L, Esvelt KM, Aach J, Guell M, DiCarlo JE, Norville JE, Church GM. 2013. RNA-guided human genome engineering via Cas9. Science 339(6121):823-826.

Marshall E. 1999. Gene therapy death prompts review of adenovirus vector. Science 286(5448):2244-2245. 
Miller JC, Holmes MC, Wang J, Guschin DY, Lee YL, Rupniewski I, Beausejour CM, Waite AJ, Wang NS, Kim KA, Gregory PD, Pabo CO, Rebar EJ. 2007. An improved zinc-finger nuclease architecture for highly specific genome editing. Nat Biotechnol 25(7):778-785.

Miller JC, Tan S, Qiao G, Barlow KA, Wang J, Xia DF, Meng X, Paschon DE, Leung E, Hinkley SJ, Dulay GP, Hua KL, Ankoudinova I, Cost GJ, Urnov FD, Zhang HS, Holmes MC, Zhang L, Gregory PD, Rebar EJ. 2011. A TALE nuclease architecture for efficient genome editing. Nat Biotechnol 29(2):143-148.

Moscou MJ, Bogdanove AJ. 2009. A simple cipher governs DNA recognition by TAL effectors. Science 326(5959):1501.

Mussolino C, Cathomen T. 2012. TALE nucleases: Tailored genome engineering made easy. Curr Opin Biotechnol 23(5):644-6450.

Mussolino C, Morbitzer R, Lutge F, Dannemann N, Lahaye T, Cathomen T. 2011. A novel TALE nuclease scaffold enables high genome editing activity in combination with low toxicity. Nucleic Acids Res 39(21):9283-9293.

Osborn MJ, Starker CG, McElroy AN, Webber BR, Riddle MJ, Xia L, Defeo AP, Gabriel R, Schmidt M, Von Kalle C, Carlson DF, Maeder ML, Joung JK, Wagner JE, Voytas DF, Blazar BR, Tolar J. 2013. TALEN-based gene correction for epidermolysis bullosa. Mol Ther 21(6):1151-1159.

Pattanayak V, Ramirez CL, Joung JK, Liu DR. 2011. Revealing off-target cleavage specificities of zinc-finger nucleases by in vitro selection. Nat Methods 8(9):765-770.

Perez-Pinera P, Ousterout DG, Gersbach CA. 2012. Advances in targeted genome editing. Curr Opin Chem Biol 16(3-4):268-277.

Reyon D, Tsai SQ, Khayter C, Foden JA, Sander JD, Joung JK. 2012. FLASH assembly of TALENs for high-throughput genome editing. Nat Biotechnol 30(5):460-465.
Silva G, Poirot L, Galetto R, Smith J, Montoya G, Duchateau P, Paques F. 2011. Meganucleases and other tools for targeted genome engineering: Perspectives and challenges for gene therapy. Curr Gene Ther 11(1):1127.

Streubel J, Blucher C, Landgraf A, Boch J. 2012. TAL effector RVD specificities and efficiencies. Nat Biotechnol 30(7):593-595.

Sun N, Zhao H. 2013a. Seamless correction of the sickle cell disease mutation of the $\mathrm{HBB}$ gene in human induced pluripotent stem cells using TALENs. Biotechnol Bioeng doi: 10.1002/bit.25018

Sun N, Zhao H. 2013b. Transcription activator-like effector nucleases (TALENs): A highly efficient and versatile tool for genome editing. Biotechnol Bioeng 110(7):1811-1821.

Sun N, Abil Z, Zhao H. 2012a. Recent advances in targeted genome engineering in mammalian systems. Biotechnol J 7(9):10741087.

Sun N, Liang J, Abil Z, Zhao H. 2012b. Optimized TAL effector nucleases (TALENs) for use in treatment of sickle cell disease. Mol Biosyst 8(4):1255-1263.

Szczepek M, Brondani V, Buchel J, Serrano L, Segal DJ, Cathomen T. 2007. Structure-based redesign of the dimerization interface reduces the toxicity of zinc-finger nucleases. Nat Biotechnol 25(7): 786-793.

\section{Supporting Information}

Additional supporting information may be found in the online version of this article at the publisher's web-site.< 